\title{
Avaliação da simetria da imagem do ramo da mandíbula em radiografias panorâmicas
}

\section{Evaluation of the symmetry of the image of the mandibular ramus in panoramic radiography}

\author{
Antonio Augusto Ferreira CARVALHO*
}

\begin{abstract}
CARVAlHO, A. A. F. Avaliação da simetria da imagem do ramo da mandíbula em radiografias panorâmicas. Pesqui Odontol Bras, v. 14, n. 3, p. 248-255, jul./set. 2000.

Os métodos radiográficos panorâmicos apresentam ampliação das imagens de estruturas anatômicas da face, com variação de uma região para outra. Atualmente, este tipo de técnica tem sido utilizado para mensurações verticais, horizontais e angulares. A área e o perímetro da imagem bilateral do ramo da mandíbula foram medidos em radiografias panorâmicas obtidas pelo sistema elipsopantomográfico, de crianças dos dois sexos, com idade cronológica entre 8 e 10 anos. Os desenhos, em papel vegetal, da imagem de cada ramo foram capturados através de "scanner" e as medidas foram feitas em microcomputador com aplicativo específico. Duas formas de separar a imagem do ramo da mandíbula, bilateralmente, foram propostas: no gônio e através de uma tangente à parte mais profunda da borda anterior da imagem do ramo que tocava a base. Os resultados obtidos permitiram evidenciar que houve simetria entre os lados, expressa pelas medidas de área e perímetro das imagens do ramo da mandíbula.
\end{abstract}

UNITERMOS: Mandíbula; Radiografia panorâmica.

\section{INTRODUÇÃO}

Em 1949, o princípio da tomografia foi adaptado para superficies curvas, surgindo então a radiografia rotacional panorâmica, método em que, usando-se apenas um filme, é obtida a imagem da maxila e da mandíbula ${ }^{1}$. Hoje, a radiografia panorâmica é um meio largamente utilizado com o propósito de auxílio diagnóstico em Odontologia e uma das principais vantagens desta técnica é que ela proporciona uma ampla observação radiográfica dos arcos dentais e estruturas associadas. Devido à facilidade de observarmos a mandíbula como um todo, incluindo os dois ramos, em uma única tomada, é possivel compararmos as duas articulações temporomandibulares, no que se refere a morfologia geral e a mudanças estruturais que nelas possam ocorrer ${ }^{1,3,9,15,16}$. Movimento do filme, relação fonte/zona tomográfica, direção do feixe e distância objeto/filme são fatores de distorção por ampliação da imagem nessas radiografias ${ }^{3,5}$.

Uma das razões para a resposta não previsivel da mandíbula ao tratamento ortopédico pode estar relacionada à complexa morfologia do osso, que pode ser dividido em quatro componentes funcio- nais: o côndilo, o ramo, o corpo e o alvéolo dental e, para que se possa compreender como ela cresce, cada componente necessita ser estudado ${ }^{8}$. A mandíbula está inter-relacionada com o crescimento do restante do complexo crânio-facial, de certa forma adaptando-se às mudanças no comportamento espacial das estruturas vizinhas ${ }^{10,17} \mathrm{e}$, depois da contribuição de cada parte ser avaliada, o profissional pode começar a determinar a melhor maneira de orientar o crescimento mandibular ${ }^{8}$.

Pantomogramas têm sido usados, tanto em elipso quanto em ortopantomografias, para avaliação do crescimento da maxila e da mandíbu$1 a^{6,14,18,19}$. Pela observação minuciosa de uma radiografia panorâmica, pode-se constatar que existe diferença no tamanho e na forma de estruturas correspondentes do lado direito e do esquerdo. Ainda que este fenômeno possa ser particularmente explicado por um inevitável efeito de distorção, típico em radiografia panorâmica rotacional, uma outra razão adicional muito importante é o fato de que existe uma assimetria esquelética fisiológica bilateral ${ }^{16,20}$. Ainda que não medindo diferenças no comprimento mandibular, WOO ${ }^{20}$ (1931) afirmou que os ossos do lado direito do crânio eram maio-

\footnotetext{
* Professor Assistente Doutor da Disciplina de Radiologia da Faculdade de Odontologia de Araçatuba da UNESP.
} 
CARVALHO, A. A. F. Avaliação da simetria da imagem do ramo da mandíbula em radiografias panorâmicas. Pesqui Odontol Bras, v. 14 , n. 3, p. $248-255$, jul./set. 2000.

res do que os do esquerdo, relacionando a assimetria da face a uma demanda funcional do aparelho mastigatório e do sistema músculo-esquelético. $\operatorname{MELNIK}^{13}$ (1992) evidenciou o fato de que há impacto da musculatura facial sobre o desenvolvimento esquelético e desarmonias dentais.

Com a utilização de arcos metálicos verticais e horizontais, adaptados em indivíduos adultos que se submeteram a radiografias panorâmicas em elipsopantomógrafo (Funk X-15) e em ortopantomógrafo (Palomex OY), ALMEIDA et al. ${ }^{1}$ (1995) verificaram que existiu diferença estatística entre a imagem real e a imagem radiográfica, apresentando-se a primeira sempre menor. Em relação aos métodos, na elipsopantomografia a ampliação apresentou-se menor, porém não estatisticamente significativa ${ }^{4}$. Avaliando a precisão de mensurações dimensionais da mandíbula feitas em ortopantomógrafo, AMIR et al. ${ }^{3}$ (1998) determinaram a confiabilidade da técnica, concluindo que é possível usar a imagem obtida neste aparelho para medidas verticais, horizontais e angulares feitas em um dos lados da mandíbula.

MADEIRA $^{12}$ (1997), em uma visão lateral do crânio, descreve o ramo como que assemelhado a um retângulo, cujas bordas posterior e inferior se encontram no ângulo da mandibula, onde se distingue a tuberosidade massetérica. LIN et al. ${ }^{10}$ (1992) descrevem, em um estudo com radiografias cefalométricas de crânios secos, que os planos mandibular e oclusal foram usados como referência, representando as bordas inferior e superior do corpo mandibular, respectivamente. Na borda inferior, o corpo da mandíbula continua-se com o ramo na altura da incisura pré-goníaca. Por essa razão, o plano mandibular é o plano de referência mais usual, tanto da borda inferior do corpo quanto do ramo. De acordo com Du BRUL ${ }^{7}$ (1980), a borda inferior, entre o ramo e o corpo, é comum na altura da incisura pré-goníaca. O ponto de intersecção entre o plano oclusal e a borda anterior do ramo é considerado como sendo o ponto no bordo superior entre as duas estruturas ${ }^{10}$.

Assim, foi propósito deste trabalho avaliar a simetria da imagem bilateral do ramo da mandíbula, medindo-se os lados direito e esquerdo em radiografias panorâmicas obtidas em elipsopantomógrafo.

\section{MATERIAL E MÉTODOS}

Foram objetos deste estudo 145 escolares brasileiros, dos dois sexos, cuja idade cronológica variou entre 8 e 10 anos, selecionados entre os matriculados para tratamento na Faculdade de Odontologia de Araçatuba - UNESP. Estes foram divididos em 3 grupos ( $\mathrm{G}$ I $=8$ anos, $\mathrm{G}$ II $=9$ anos e $\mathrm{G}$ III $=10$ anos).

Para o exame radiográfico, utilizamos a técnica panorâmica, obtida em aparelho Funk X-15, chassi plástico flexível montado com placas intensificadoras e filmes Kodak.

Com os filmes processados e secos, colocamos papel vegetal sobre as radiografias em negatoscópio e desenhamos o ramo da mandíbula de duas formas (Figura 1), dividindo-o: 1. exatamente no ângulo goniaco (corte gônio), obtido através da bissetriz formada pela imagem do plano mandibular e por uma tangente ao bordo posterior da imagem do ramo; 2. traçando uma tangente ao ponto mais profundo da concavidade anterior da imagem do ramo, até tocar a base da mandíbula (corte bordo anterior). Tomamos os desenhos e capturamos sua
FIGURA 1 - Desenho esquemático de uma radiografia panorâmica contendo as formas de divisão do ramo da mandíbula no gônio e no bordo anterior.

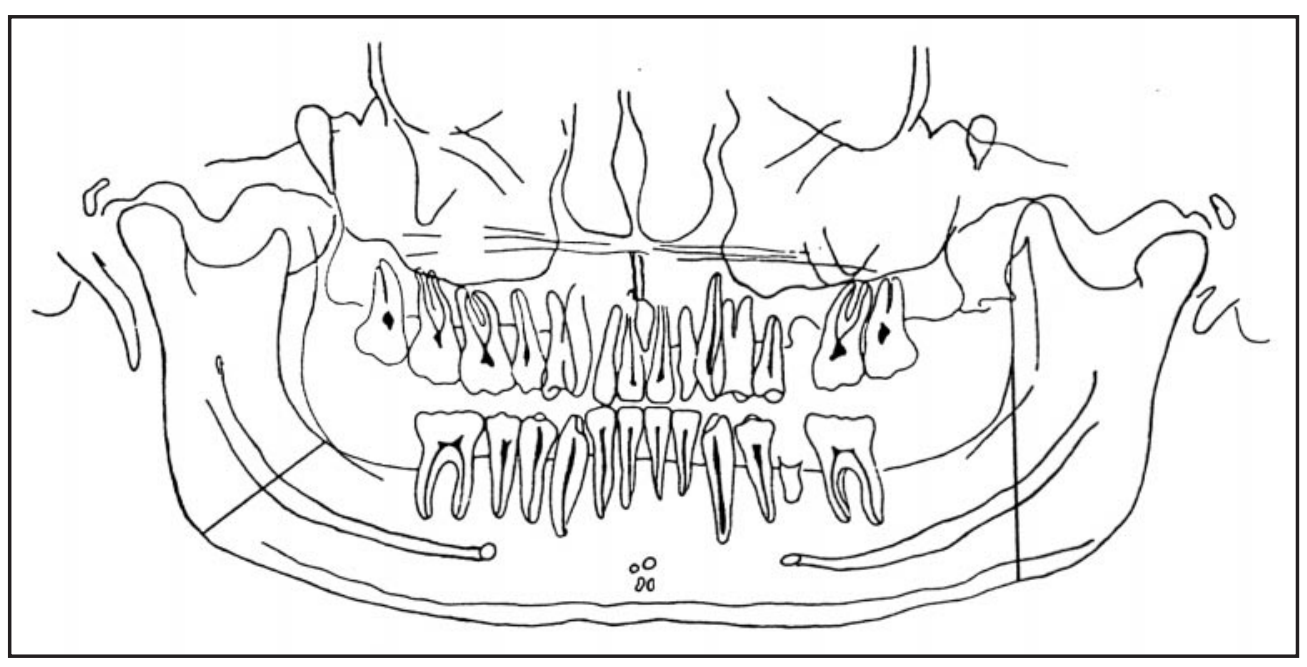


CARVAlHO, A. A. F. Avaliação da simetria da imagem do ramo da mandíbula em radiografias panorâmicas. Pesqui Odontol Bras, v. 14 , n. 3, p. 248-255, jul./set. 2000.

TABELA 1 - Média e erro padrão da média das medidas de área das imagens do ramo da mandíbula (D = lado direito; $\mathrm{E}=$ lado esquerdo) em milimetros ${ }^{2}$.

\begin{tabular}{|c|c|c|c|c|c|c|c|c|c|c|c|}
\hline \multicolumn{12}{|c|}{ Corte gônio } \\
\hline \multicolumn{6}{|c|}{ Sexo masculino } & \multicolumn{6}{|c|}{ Sexo feminino } \\
\hline \multicolumn{2}{|c|}{ Grupo I $(n=24)$} & \multicolumn{2}{|c|}{ Grupo II $(\mathrm{n}=22)$} & \multicolumn{2}{|c|}{ Grupo III $(\mathrm{n}=21)$} & \multicolumn{2}{|c|}{ Grupo I $(\mathrm{n}=30)$} & \multicolumn{2}{|c|}{ Grupo II $(\mathrm{n}=21)$} & \multicolumn{2}{|c|}{ Grupo III $(n=28)$} \\
\hline $\mathrm{D}$ & $\mathrm{E}$ & $\mathrm{D}$ & $\mathrm{E}$ & $\mathrm{D}$ & $\mathrm{E}$ & $\mathrm{D}$ & $\mathrm{E}$ & $\mathrm{D}$ & $\mathrm{E}$ & $\mathrm{D}$ & $\mathrm{E}$ \\
\hline $1.297,1$ & 1.405 & $1.313,1$ & $1.342,1$ & $1.487,2$ & $1.558,6$ & $1.276,7$ & $1.357,6$ & $1.306,7$ & $1.372,9$ & $1.329,1$ & $1.408,4$ \\
\hline 30,97 & 34,09 & 32,28 & 34,15 & 35,77 & 37,88 & 26,42 & 22,47 & 31 & 28,45 & 27,06 & 30,58 \\
\hline \multicolumn{12}{|c|}{ Corte bordo anterior } \\
\hline \multicolumn{6}{|c|}{ Sexo masculino } & \multicolumn{6}{|c|}{ Sexo feminino } \\
\hline \multicolumn{2}{|c|}{ Grupo I $(n=24)$} & \multicolumn{2}{|c|}{ Grupo II $(n=22)$} & \multicolumn{2}{|c|}{ Grupo III $(n=21)$} & \multicolumn{2}{|c|}{ Grupo I $(n=30)$} & \multicolumn{2}{|c|}{ Grupo II $(\mathrm{n}=21)$} & \multicolumn{2}{|c|}{ Grupo III $(\mathrm{n}=28)$} \\
\hline $\mathrm{D}$ & $\mathrm{E}$ & $\mathrm{D}$ & $\mathrm{E}$ & $\mathrm{D}$ & $\mathrm{E}$ & $\mathrm{D}$ & $\mathrm{E}$ & $\mathrm{D}$ & $\mathrm{E}$ & $\mathrm{D}$ & $\mathrm{E}$ \\
\hline $1.840,9$ & $2.038,3$ & $1.852,4$ & $1.921,8$ & $2.122,8$ & $2.211,1$ & $1.784,9$ & $1.938,6$ & $1.866,2$ & $1.969,2$ & $1.915,1$ & $1.985,2$ \\
\hline 44,89 & 63,14 & 42,21 & 51,24 & 40,68 & 49,33 & 45,85 & 43,79 & 60,14 & 53,03 & 48,44 & 47,51 \\
\hline
\end{tabular}

TABELA 2 - Média e erro padrão da média das medidas de perímetro das imagens do ramo da mandíbula (D = lado direito; $\mathrm{E}$ = lado esquerdo) em milimetros.

\begin{tabular}{|c|c|c|c|c|c|c|c|c|c|c|c|}
\hline \multicolumn{12}{|c|}{ Corte gônio } \\
\hline \multicolumn{6}{|c|}{ Sexo masculino } & \multicolumn{6}{|c|}{ Sexo feminino } \\
\hline \multicolumn{2}{|c|}{ Grupo I $(n=24)$} & \multicolumn{2}{|c|}{ Grupo II $(n=22)$} & \multicolumn{2}{|c|}{ Grupo III $(\mathrm{n}=21)$} & \multicolumn{2}{|c|}{ Grupo I $(n=30)$} & \multicolumn{2}{|c|}{ Grupo II $(n=21)$} & \multicolumn{2}{|c|}{ Grupo III $(\mathrm{n}=28)$} \\
\hline $\mathrm{D}$ & $\mathrm{E}$ & $\mathrm{D}$ & $\mathrm{E}$ & $\mathrm{D}$ & E & $\mathrm{D}$ & $\mathrm{E}$ & $\mathrm{D}$ & E & $\mathrm{D}$ & $\mathrm{E}$ \\
\hline 183,87 & 191,61 & 189,22 & 192,44 & 205,65 & 206,9 & 184,45 & 191,41 & 192,86 & 199,05 & 199,37 & 198,65 \\
\hline 2,35 & 2,61 & 2,3 & 2,32 & 3,83 & 2,88 & 2,08 & 1,59 & 3,22 & 3,74 & 3,24 & 2,23 \\
\hline \multicolumn{12}{|c|}{ Corte bordo anterior } \\
\hline \multicolumn{6}{|c|}{ Sexo masculino } & \multicolumn{6}{|c|}{ Sexo feminino } \\
\hline \multicolumn{2}{|c|}{ Grupo I $(\mathrm{n}=24)$} & \multicolumn{2}{|c|}{ Grupo II $(n=22)$} & \multicolumn{2}{|c|}{ Grupo III $(\mathrm{n}=21)$} & \multicolumn{2}{|c|}{ Grupo I $(n=30)$} & \multicolumn{2}{|c|}{ Grupo II $(n=21)$} & \multicolumn{2}{|c|}{ Grupo III $(\mathrm{n}=28)$} \\
\hline $\mathrm{D}$ & $\mathrm{E}$ & $\mathrm{D}$ & $\mathrm{E}$ & $\mathrm{D}$ & $\mathrm{E}$ & $\mathrm{D}$ & $\mathrm{E}$ & $\mathrm{D}$ & $\mathrm{E}$ & $\mathrm{D}$ & $\mathrm{E}$ \\
\hline 223,74 & 227,48 & 225,6 & 229,75 & 243,76 & 246,22 & 229,1 & 234,76 & 235,19 & 246,8 & 247,66 & 254,7 \\
\hline 3,48 & 2,87 & 2,95 & 3,16 & 4,26 & 3,12 & 3,61 & 3,15 & 4,37 & 4,85 & 4,19 & 3,82 \\
\hline
\end{tabular}

imagem com a utilização de "scanner" (Hewlett Packard ScanJet IIcx) acoplado a microcomputador IBM PC. Com a utilização de um aplicativo para análise de imagens (Jandel Sigma Scan), medimos a área e o perímetro das imagens bilaterais do ramo da mandíbula. A calibração para as medidas foi executada, repetidamente, em cada momento de medida, para aumentarmos a confiabilidade, uma vez que o tamanho e a forma da imagem na tela do monitor podia interferir na utilização do aplicativo.

Após a realização de todas as medidas, os resultados foram submetidos a tratamento estatístico, quando utilizamos o teste $t$ de Student para dados não-pareados e obtivemos os coeficientes de correlação entre os valores de cada parâmetro e entre os lados direito e esquerdo do mesmo parâmetro.

\section{RESULTADOS}

Podemos observar (pelas Tabelas e Quadros) que, ao medirmos a imagem do ramo da mandíbula através do corte no gônio, não houve diferença estatística entre os valores de área, entre os lados, nos dois sexos (Tabela 1) não houve diferença entre os valores de perimetro, entre os lados, nos dois 
CARVALHO, A. A. F. Avaliação da simetria da imagem do ramo da mandíbula em radiografias panorâmicas. Pesqui Odontol Bras, v. 14 , n. 3, p. $248-255$, jul./set. 2000.

sexos (Tabela 2). Quando exprimimos os valores como a média dos dois lados, tanto de área quanto de perimetro, observamos valores significativamente maiores no sexo masculino no grupo III (Tabelas 3 e 4); existiu forte correlação entre os valores de área e entre os de perímetro, nos dois sexos nos 3 grupos (Quadros 1 e 2); houve forte correlação entre os valores de área e perímetro nos 3 grupos nos dois sexos (Quadro 3).

Quando medimos o ramo através do corte no bordo anterior até a base da mandibula observamos que não houve diferença estatística entre os valores de área nem entre os de perímetro nos lados direito e esquerdo, nos dois sexos (Tabelas 1 e 2). Quando exprimimos os valores como a média dos dois lados, tanto da área quanto de perímetro, observamos que: houve diferença entre os sexos, sendo os valores de área, do sexo masculino, esta- tisticamente superiores no grupo III (Tabela 3); os coeficientes de correlação entre os valores de área e de perimetro foram estatisticamente significativos quando comparamos os lados nos dois sexos nos 3 grupos (Quadros 1 e 2); houve forte correlação entre os valores de área e perímetro, nas duas formas de medida da imagem do ramo da mandíbula nos três grupos, nos dois sexos (Quadro 3).

\section{DISCUSSÃO}

Os resultados obtidos na técnica panorâmica dependem da qualidade da imagem e do conhecimento técnico do profissional, e alguns aspectos estão envolvidos: subjetivos, se relacionados ao profissional quando da interpretação radiográfica; objetivos quando envolvem a qualidade da imagem, estando relacionados à distorção, borramento e artefatos. Há um grande número de aparelhos

TABELA 3 - Média e erro padrão da média das medidas de área (lado direito + esquerdo) das imagens do ramo da mandíbula em milimetros ${ }^{*}{ }^{*}=\mathrm{p}<0,01$ em relação ao sexo feminino).

\begin{tabular}{|c|c|c|c|c|c|}
\hline \multicolumn{6}{|c|}{ Corte gônio } \\
\hline \multicolumn{3}{|c|}{ Sexo masculino } & \multicolumn{3}{|c|}{ Sexo feminino } \\
\hline Grupo I $(n=24)$ & Grupo II $(\mathrm{n}=22)$ & Grupo III $(\mathrm{n}=21)$ & Grupo I $(n=30)$ & Grupo II $(n=21)$ & Grupo III $(\mathrm{n}=28)$ \\
\hline $1.351,1$ & $1.328,0$ & $1.523,1^{*}$ & $1.318,0$ & $1.339,8$ & $1.368,8$ \\
\hline 31,14 & 31,85 & 34,37 & 22,33 & 28,12 & 26,95 \\
\hline \multicolumn{6}{|c|}{ Corte bordo anterior } \\
\hline \multicolumn{3}{|c|}{ Sexo masculino } & \multicolumn{3}{|c|}{ Sexo feminino } \\
\hline Grupo I $(n=24)$ & Grupo II $(\mathrm{n}=22)$ & Grupo III $(\mathrm{n}=21)$ & Grupo I $(n=30)$ & Grupo II $(n=21)$ & Grupo III $(\mathrm{n}=28)$ \\
\hline $1.939,6$ & $1.887,1$ & $2.166,0^{*}$ & $1.861,8$ & $1.917,7$ & $1.950,1$ \\
\hline 51,7 & 43,8 & 41,29 & 42,05 & 54,43 & 42,78 \\
\hline
\end{tabular}

TABELA 4 - Média e erro padrão da média das medidas de perímetro (lado direito + esquerdo) das imagens do ramo da mandíbula em milimetros $\left(^{*}=\mathrm{p}<0,01\right.$ em relação ao sexo masculino).

\begin{tabular}{|c|c|c|c|c|c|}
\hline \multicolumn{6}{|c|}{ Corte gônio } \\
\hline \multicolumn{3}{|c|}{ Sexo masculino } & \multicolumn{3}{|c|}{ Sexo feminino } \\
\hline Grupo I $(n=24)$ & Grupo II $(n=22)$ & Grupo III $(\mathrm{n}=21)$ & Grupo I $(\mathrm{n}=30)$ & Grupo II $(\mathrm{n}=21)$ & Grupo III $(\mathrm{n}=28)$ \\
\hline 187,74 & 190,83 & 206,28 & 187,86 & 195,96 & 199,01 \\
\hline 2,37 & 2,04 & 2,9 & 1,63 & 3,21 & 2,51 \\
\hline \multicolumn{6}{|c|}{ Corte bordo anterior } \\
\hline \multicolumn{3}{|c|}{ Sexo masculino } & \multicolumn{3}{|c|}{ Sexo feminino } \\
\hline Grupo I $(n=24)$ & Grupo II $(n=22)$ & Grupo III $(\mathrm{n}=21)$ & Grupo I $(n=30)$ & Grupo II $(n=21)$ & Grupo III $(\mathrm{n}=28)$ \\
\hline 225,61 & 227,68 & 244,99 & 231,93 & $235,19^{*}$ & 251,18 \\
\hline 2,8 & 2,8 & 3,41 & 2,64 & 4,37 & 3,19 \\
\hline
\end{tabular}


CARVAlHO, A. A. F. Avaliação da simetria da imagem do ramo da mandíbula em radiografias panorâmicas. Pesqui Odontol Bras, v. 14 , n. 3, p. 248-255, jul./set. 2000.

para obtenção de radiografias panorâmicas e o fator de ampliação varia conforme o fabricante, devido à diferença na projeção geométrica utilizada ${ }^{3}$. No método panorâmico, obtém-se uma imagem bidimensional de um objeto tridimensional e suas medidas foram obtidas, neste estudo, sobre traçado, com certa margem de erro. Para compensar os posicionamentos assimétricos de alguns crânios de indivíduos, CARVALHO ${ }^{5}$ (1993) obteve médias aritméticas das distâncias bilaterais homólogas, para correção de possiveis distorções em lateralidade.
A distorção da imagem obtida na radiografia panorâmica refere-se a uma ampliação desigual entre as dimensões das imagens nos sentidos vertical e horizontal, sendo o posicionamento incorreto do paciente um dos principais fatores responsáveis pela ocorrência das distorções. Essas radiografias estão caracterizadas por diferentes fatores de ampliação para as dimensões vertical e horizontal e estes fatores, nas duas dimensões, são iguais para aquelas estruturas situadas na zona tomográfica ${ }^{6}$. Na técnica panorâmica, a distância focal, em conjunto com a distância entre área focal/plano tomográfico, determinam a ampliação da imagem; no

QUADRO 1 - Coeficientes de correlação entre os valores de área das imagens do ramo da mandíbula ( $\mathrm{D}$ = lado direito; $\mathrm{E}=$ lado esquerdo).

\begin{tabular}{|c|c|c|c|c|c||}
\hline \multicolumn{5}{|c|}{ Sexo masculino } & \multicolumn{3}{c||}{ Sexo feminino } \\
\hline \multicolumn{7}{|c|}{ Grupo II } & Grupo III & Grupo I & Grupo II & Grupo III \\
\hline Grupo I & D x E & D x E & D x E & D x E & D x E \\
\hline $\mathrm{D} \times \mathrm{E}$ & $\mathrm{r}=0,84$ & $\mathrm{r}=0,74$ & $\mathrm{r}=0,74$ & $\mathrm{r}=0,80$ & $\mathrm{r}=0,75$ \\
\hline $\mathrm{r}=0,83$ & $\mathrm{p}<0,0001$ & $\mathrm{p}=0,0001$ & $\mathrm{p}<0,0001$ & $\mathrm{p}<0,0001$ & $\mathrm{p}<0,0001$ \\
\hline $\mathrm{p}<0,0001$ & Corte bordo anterior & Sexo feminino \\
\hline \multicolumn{7}{|c|}{ Sexo masculino } \\
\hline Grupo I & Grupo II & Grupo III & Grupo I & Grupo II & Grupo III \\
\hline $\mathrm{D} \times \mathrm{E}$ & $\mathrm{D} \times \mathrm{E}$ & $\mathrm{D} \times \mathrm{E}$ & $\mathrm{D} \times \mathrm{E}$ & $\mathrm{D} \times \mathrm{E}$ & $\mathrm{D} \times \mathrm{E}$ \\
\hline $\mathrm{r}=0,84$ & $\mathrm{r}=0,75$ & $\mathrm{r}=0,68$ & $\mathrm{r}=0,76$ & $\mathrm{r}=0,85$ & $\mathrm{r}=0,59$ \\
\hline $\mathrm{p}<0,0001$ & $\mathrm{p}<0,0001$ & $\mathrm{p}=0,0007$ & $\mathrm{p}<0,0001$ & $\mathrm{p}<0,0001$ & $\mathrm{p}=0,0012$ \\
\hline
\end{tabular}

QUADRO 2 - Coeficientes de correlação entre os valores de perímetro das imagens do ramo da mandíbula (D = lado direito; $\mathrm{E}=$ lado esquerdo).

\begin{tabular}{|c|c|c|c|c|c||}
\hline \multicolumn{5}{|c|}{ Corte gônio } \\
\hline \multicolumn{7}{|c|}{ Sexo masculino } & \multicolumn{3}{c||}{ Sexo feminino } \\
\hline Grupo I & Grupo II & Grupo III & Grupo I & Grupo II & Grupo III \\
\hline $\mathrm{D} \times \mathrm{E}$ & $\mathrm{D} \times \mathrm{E}$ & $\mathrm{D} \times \mathrm{E}$ & $\mathrm{D} \times \mathrm{E}$ & $\mathrm{D} \times \mathrm{E}$ & $\mathrm{D} \times \mathrm{E}$ \\
\hline $\mathrm{r}=0,83$ & $\mathrm{r}=0,62$ & $\mathrm{r}=0,79$ & $\mathrm{r}=0,60$ & $\mathrm{r}=0,70$ & $\mathrm{r}=0,67$ \\
\hline $\mathrm{p}<0,0001$ & $\mathrm{p}=0,002$ & $\mathrm{p}<0,0001$ & $\mathrm{p}=0,0004$ & $\mathrm{p}=0,0004$ & $\mathrm{p}<0,0001$ \\
\hline \multicolumn{7}{|c||}{ Corte bordo anterior } \\
\hline \multicolumn{7}{|c|}{ Sexo masculino } & Grupo I & Grupo II & Grupo III \\
\hline Grupo I & Grupo II & Grupo III & $\mathrm{D} \times \mathrm{E}$ & $\mathrm{D} \times \mathrm{E}$ & $\mathrm{D} \times \mathrm{E}$ \\
\hline $\mathrm{D} \times \mathrm{E}$ & $\mathrm{D} \times \mathrm{E}$ & $\mathrm{D} \times \mathrm{E}$ & $\mathrm{r}=0,22$ & $\mathrm{r}=0,56$ & $\mathrm{r}=0,26$ \\
\hline $\mathrm{r}=0,55$ & $\mathrm{r}=0,72$ & $\mathrm{r}=0,70$ & $\mathrm{p}=0,007$ & \\
\hline $\mathrm{p}=0,004$ & $\mathrm{p}=0,0001$ & $\mathrm{p}=0,0004$ & & \\
\hline
\end{tabular}


CARVALHO, A. A. F. Avaliação da simetria da imagem do ramo da mandíbula em radiografias panorâmicas. Pesqui Odontol Bras, v. 14 , n. 3, p. 248-255, jul./set. 2000.

QUADRO 3 - Coeficientes de correlação entre os valores de área e perímetro (lado direito + esquerdo; SM = sexo masculino, $\mathrm{SF}=$ sexo feminino; $\mathrm{AGo}=$ área corte gônio, $\mathrm{PGo}=$ perímetro corte gônio, $\mathrm{ABa}=$ área corte bordo anterior, $\mathrm{PBa}=$ perimetro corte bordo anterior).

\begin{tabular}{|c|c|c|c|}
\hline \multicolumn{2}{|c|}{ Grupo I } & \multicolumn{2}{|c|}{ Grupo I } \\
\hline SM & SF & SM & SF \\
\hline AGo x PGo & AGo x PGo & $\mathrm{ABa} \times \mathrm{PBa}$ & $\mathrm{ABa} \times \mathrm{PBa}$ \\
\hline $0,89(\mathrm{p}<0,0001)$ & $0,80(\mathrm{p}<0,0001)$ & $0,89(\mathrm{p}<0,0001)$ & $0,68(\mathrm{p}<0,0001)$ \\
\hline \multicolumn{2}{|c|}{ Grupo II } & \multicolumn{2}{|c|}{ Grupo II } \\
\hline SM & $\mathrm{SF}$ & SM & $\mathrm{SF}$ \\
\hline AGo x PGo & AGo x PGo & $\mathrm{ABa} \times \mathrm{PBa}$ & $\mathrm{ABa} \times \mathrm{PBa}$ \\
\hline $0,90(\mathrm{p}<0,0001)$ & $0,76(\mathrm{p}<0,0001)$ & $0,44(p=0,04)$ & $0,67(\mathrm{p}=0,0007)$ \\
\hline \multicolumn{2}{|c|}{ Grupo III } & \multicolumn{2}{|c|}{ Grupo III } \\
\hline SM & $\mathrm{SF}$ & $\mathrm{SM}$ & $\mathrm{SF}$ \\
\hline AGo x PGo & AGo x PGo & $\mathrm{ABa} \times \mathrm{PBa}$ & $\mathrm{ABa} \times \mathrm{PBa}$ \\
\hline $0,67(\mathrm{p}=0,0008)$ & $0,60(p=0,0006)$ & $0,71(\mathrm{p}=0,0002)$ & $0,30(\mathrm{p}=0,12)$ \\
\hline
\end{tabular}

elipsopantomógrafo, estas distâncias são constantes, resultando em uma ampliação vertical uniforme, em toda a radiografia, ao redor de $19 \%{ }^{2}$, enquanto AMIR et al. ${ }^{3}$ observaram índices variados de ampliação para diferentes regiões da mandíbula, utilizando ortopantomógrafo.

Os resultados obtidos por ALMEIDA et al. ${ }^{1}$ (1995) permitiram afirmar que as imagens radiográficas horizontais dos segmentos de arcos oclusais, em elipsopantomógrafo, apresentaram uma distorção nos segmentos posteriores, tanto na maxila quanto na mandibula, bastante homogênea. Esses resultados foram menores e mais homogêneos do que aqueles obtidos em ortopantomógrafo. Encontraram também uma equivalência entre os resultados das imagens do lado direito e esquerdo, devido ao fato de uma correta centralização do plano sagital mediano dos indivíduos nos aparelhos. LARHEIM; SVANAES ${ }^{9}$ (1986), avaliando a exatidão de dimensões lineares e angulares em ortopantomografias, afirmaram que não existiu diferença entre os lados direito e esquerdo, indicando que a fronte foi simetricamente posicionada no aparelho com o auxílio de um indicador luminoso na linha mediana da face.

CAHALI et al. ${ }^{4}$ (1991), estudando as variações dimensionais lineares horizontais e verticais dos seios maxilares em radiografias panorâmicas em elipso e ortopantomógrafo, observaram que, para todas as dimensões estudadas, relacionadas aos lados direito e esquerdo, não houve diferença estatística significativa. Quando existe diferença entre os lados, isto se deve ao incorreto posicionamento da cabeça do paciente nos aparelhos pantomográficos, à movimentação do paciente no momento da tomada, a uma assimetria do próprio aparelho relacionada ao princípio de obtenção da radiografia ou ainda a uma assimetria da face ${ }^{4,11}$. AMIR et al. ${ }^{3}$ (1998) concordam com essa afirmação, acrescentando que o fator de ampliação pode variar de um aparelho para outro, mesmo que tenha sido produzido pelo mesmo fabricante e, por isso, é essencial o uso de um mesmo aparelho em estudos longitudinais.

Trabalhando com distorção da imagem em radiografias panorâmicas obtidas em ortopantomógrafo, ROWSE ${ }^{15}$ (1971) observou graus de ampliação diferentes em várias partes da mandíbula, o que também foi observado por AMIR et al. ${ }^{3}$ (1998), em razão da alteração área focal-filme-paciente. As regiões que apresentaram menor distorção horizontal, na imagem da mandíbula, foram as do processo coronóide/côndilo e do bordo anterior na sinfise. Observou-se também que houve menor distorção vertical na região que vai do ângulo da mandíbula ao côndilo ${ }^{15}$.

De acordo com nossos resultados, a radiografia panorâmica obtida em elipsopantomógrafo forneceu uma imagem completa de toda a mandíbula, livre de superposição de outras imagens de estruturas anatômicas na zona tomográfica, proporcionando excelente meio para mensurações. Apesar da distorção, inevitável na opinião de TÜRP et al. ${ }^{16}$ 
CARVAlHO, A. A. F. Avaliação da simetria da imagem do ramo da mandíbula em radiografias panorâmicas. Pesqui Odontol Bras, v. 14 , n. 3, p. 248-255, jul./set. 2000.

(1995), que resulta em ampliação das imagens observadas, o uso do mesmo aparelho pelo mesmo operador com os cuidados técnicos para posicionamento dos indivíduos, permitiu uma uniformidade de resultados (os mesmos índices de ampliação para toda a amostra) e boa visualização para avaliação do crescimento do ramo da mandíbula, através de valores numéricos. As formas de divisão da imagem do ramo mostraram-se úteis para este propósito, sendo a forma corte bordo anterior muito semelhante à sugerida por Du BRUL ${ }^{7}$ (1980) e por LIN et al. ${ }^{10}$ (1992), embora estes tenham usado radiografias cefalométricas em sua metodologia.

Os resultados das medidas de área e perímetro da imagem do ramo da mandíbula mostraram que, apesar da ampliação observada na técnica empregada e de diferenças na forma das imagens, não existiu diferença estatística entre os lados direito e esquerdo na grande maioria dos indivíduos. Isto sugeriu que houve o mesmo índice de ampliação em todas as tomadas, nas duas formas de medirmos esta região da mandíbula (Tabelas 1 e 2), o que está de acordo com os resultados de LARHEIM; SVANAES ${ }^{9}$ (1986), de CAHALI et al. ${ }^{4}$ (1991), de AMIR et al. ${ }^{3}$ (1998) e também com os de ALPERN $^{2}$ (1979), quando observou uniformidade de ampliação em toda a radiografia. Quando expressamos os valores de área como a média aritmética dos lados, observamos diferença estatística entre os sexos no grupo III nas duas formas de medida (Tabela 3), sendo o valor médio do sexo masculino maior do que o do sexo feminino, podendo estar re- lacionado ao crescimento ósseo diferenciado, entre os sexos, nesta faixa etária.

Pelo exposto nos Quadros 1 e 2, observamos correlação estatisticamente significativa entre os lados, nos três grupos, nos dois sexos, tanto nos valores de área quanto nos de perímetro, nas duas formas de medida (exceção ao perímetro no sexo feminino nos grupos I e III, corte bordo anterior, Quadro 2). Resultados estatisticamente significativos também foram observados quando correlacionamos os valores de área com os de perimetro (média aritmética dos lados) da mesma forma de medida (Quadro 3). Assim, os lados direito e esquerdo foram simétricos, quando medimos área e perímetro da imagem do ramo da mandíbula separada no gônio ou através de uma tangente à concavidade da borda anterior tocando a base e estes resultados nos permitiram afirmar que é possivel realizar medidas precisas com relação à área e perímetro da imagem do ramo da mandíbula, obtida em elipsopantomógrafo, em um dos lados, de acordo com a metodologia proposta.

\section{CONCLUSÃO}

Estes resultados permitem concluir que a radiografia panorâmica, obtida em elipsopantomógrafo Funk X-15, permitiu evidenciar simetria na imagem do ramo da mandíbula, nos lados direito e esquerdo, quando área e perímetro foram medidos em crianças dos dois sexos com idade cronológica entre 8 e 10 anos, através de imagens digitalizadas.

CARVAlHO, A. A. F. Evaluation of the symmetry of the image of the mandibular ramus in panoramic radiography.

Pesqui Odontol Bras, v. 14, n. 3, p. 248-255, jul./set. 2000.

Panoramic radiographic methods provide images which present distortions of bilateral anatomical structures of the face as well as magnification. Currently, panoramic radiographs have been used to analyze vertical, horizontal and angular measurements. In an attempt to evaluate the presence of symmetry in the bilateral images of the mandibular rami, both their area and perimeter were obtained from panoramic radiographs of 8- to 10-year old children. The contour of the images of both rami was divided at the gonial angle and at the deeper point of the anterior border and it was transferred to translucent tracing paper and then to a computer through a scanner apparatus. The images were measured using a scan software and the values obtained were analyzed. The results showed that there was symmetry in both area and perimeter measurements, in male and female children.

UNITERMS: Mandible; Radiography, panoramic.

\section{REFERÊNCIAS BIBLIOGRÁFICAS}

1. AlmeidA, S. M.; BOSCOLO, F. N.; MONTEBElo FILHO, A. Estudo das distorções da imagem radiográfica produzida em aparelhos panorâmicos que se utilizam dos princípios ortopantomográficos e elipsopantomográficos. Rev Odontol Univ São Paulo, v. 9, p. 91-99, 1995.

2. ALPERN, M. C. Analysis of panoramic cephalometrics using a skeletal cephalostat. Angle Orthod, v. 49, p. $110-120,1979$. 
CARVALHO, A. A. F. Avaliação da simetria da imagem do ramo da mandíbula em radiografias panorâmicas. Pesqui Odontol Bras, v. 14 , n. 3, p. 248-255, jul./set. 2000.

3. AMIR, C.; ASJAC, C.; MELITA, V. P. et al. Evaluation of the precision of dimensional measurements of the mandible on panoramic radiographs. Oral Surg, v. 86, p. 242-248, 1998.

4. CAHALI, J. B.; FREITAS, A.; ARAÚJO, V. C. Estudo das variações dimensionais lineares horizontais e verticais dos seios maxilares, com o emprego das radiografias panorâmicas (elipso e ortopantomografias). Rev Odontol Univ São Paulo, v. 5, p. 47-54, 1991.

5. CARVALHO, P. L. Estudo radiográfico comparativo das ampliações de segmentos ósseos crânio-faciais, em crânios, nas ortopantomografias e elipsopantomografias. São Paulo, 1993. 121 p. Tese (Doutorado) Faculdade de Odontologia, Universidade de São Paulo, 1993.

6. CAVALCANTI, M. G. P.; ARITA, E. S.; VAROLI, O. J. et al. Estudo radiográfico comparativo de grandezas lineares maxilo-mandibulares por meio de elipsopantomografias e telerradiografias frontais em leucodermas e descendentes de japoneses. Rev Odontol Univ São Paulo, v. 7, p. 115-119, 1993.

7. Du BRUL, B. L. Sicher's oral anatomy, 7. ed. St. Louis : The C. V. Mosby, 1980 apud Bull Tokyo Med Dent Univ, v. 39, p. 1-7, 1992.

8. HANS, M. G.; ENLOW, D. H.; NOACHTAR, R. Age-related in mandibular ramus growth: a histologic study. Angle Orthod, v. 65, p. 335-340, 1995.

9. LARHEIM, T. A.; SVANAES, D. B. Reproducibility of rotational panoramic radiography: mandibular linear dimensions and angles. Am J Orthod Dentofac Orthop, v. 90, p. 45-51, 1986.

10. LIN, Y. F.; ONO, V.; ONO, H. A study on a method to identify the border between mandibular corpus and ramus on lateral cephalometric radiograph. Bull Tokyo Med Dent Univ, v. 39, p. 1-7, 1992.

11. LUND, T. M.; MANSON-HING, L. R. A study of the focal troughs of three panoramic dental $\mathrm{x}$-ray machines.
Part II: image dimensions. Oral Surg Oral Med Oral Patol, v. 39, p. 647-653, 1975.

12. MADEIRA, M. C. Anatomia da face. 2. ed. São Paulo : Sarvier, 1997. 176 p.

13. MELNIK, A. K. A cephalometric study of mandibular asymmetry in a longitudinally followed sample of growing children. Am J Orthod Dentofac Orthop, v. 101, p. 355-366, 1992.

14. PANElla, J.; FREITAS, A.; VAROLI, O. J. et al. Contribuição para o estudo do dimorfismo sexual através da análise de medidas lineares da mandíbula, obtidas em pantomogramas de elipsopantomografias. Rev Odontol Univ São Paulo, v. 2, p. 92-96, 1988.

15. ROWSE, C. W. Notes on interpretation of the orthopantomogram. Brit Dent J, v. 130, p. 425-434, 1971.

16. TÜRP, J. C.; VACH, W.; HARBICH, K. W. et al. Determining mandibular condyle and ramus height with the help of an orthopantomogram - a valid method? J Oral Rehabilitation, v. 23, p. 395-400, 1996.

17. URSI, W. J. S. Crescimento e alterações nas relações mandibulares dos 6 aos 8 anos de idade. Ortodontia, v. 29, p. 4-12, 1996.

18. VAROLI, O. J.; FREITAS, A.; SILVA, M. Estudo radiográfico dos incrementos ósseos mandíbulo-faciais em qüinqüênios, de pacientes leucodermas, através de elipsopantomografias. Rev Odontol Univ São Paulo, v. 2, p. 25-31, 1988.

19. VAROLI, O. J.; FREITAS, A. Estudo radiográfico do dimorfismo sexual em leucodermas nas elipsopantomografias, por meio de avaliações lineares dos incrementos ósseos faciais. Rev Odontol Univ São Paulo, v. 5, p. 84-89, 1991.

20. WOO, T. L. On the asymmetry of human skull. Biometrika, v. 22, p. 324-352, 1931 apud Am J Orthod Dentofac Orthop, v. 101, p. 355-366, 1992. 